\title{
Interference of Bose-Einstein condensates: quantum non-local effects
}

\author{
W. J. Mullin ${ }^{a}$ and F. Laloë ${ }^{b}$ \\ ${ }^{a}$ Department of Physics, University of Massachusetts, Amherst, Massachusetts 01003 USA \\ ${ }^{b}$ Laboratoire Kastler Brossel, ENS, UPMC, CNRS; 24 rue Lhomond, 75005 Paris, France
}

\begin{abstract}
Quantum systems in Fock states do not have a phase. When two or more Bose-Einstein condensates are sent into interferometers, they nevertheless acquire a relative phase under the effect of quantum measurements. The usual explanation relies on spontaneous symmetry breaking, where phases are ascribed to all condensates and treated as unknown classical quantities. However, this image is not always sufficient: when all particles are measured, quantum mechanics predicts probabilities that are sometimes in contradiction with it, as illustrated by quantum violations of local realism. In this letter, we show that interferometers can be used to demonstrate a large variety of violations with an arbitrarily large number of particles. With two independent condensates, we find violations of the BCHSH inequalities, as well as new $N$-body Hardy impossibilities. With three condensates, we obtain new GHZ (Greenberger, Horne and Zeilinger) type contradictions.
\end{abstract}

Gaseous Bose-Einstein condensates (BEC) can be used as sources to perform experiments with atomic interferometers [1]. With a single condensate, the interference effects depend on the difference of the accumulated phases along the arms of the interferometer. With two or more BEC's, their relative phase introduces new physics into the problem. The usual view is that, when spontaneous symmetry breaking takes place at the Bose-Einstein transition, each condensates acquires a phase, with a completely random value. The outcome of a given experiment can then be obtained by assuming the existence of this initial classical phase; for an ensemble of realizations, an average over all of its possible values is necessary. Spectacular experiments with alkali atoms originating from two independent BEC have confirmed this view [2]. Long before, Anderson [3] had proposed a thought experiment raising the famous question "Do superfluids that have never seen each other have a well-defined relative phase?" The question is not trivial since, in quantum mechanics, the Bose-Einstein condensates of superfluids are naturally described by Fock states, for which the phase is completely undetermined. Nevertheless, various authors [4, 5] have shown that repeated quantum measurements of the relative phase of two Fock states make a welldefined value emerge spontaneously with a random value [6, 7]. Then, considering that the phase appears under the effect of spontaneous symmetry breaking, when the BEC's are formed, or later, under the effect of measurements, seems to be only as a matter of taste.

But a closer examination of the problem shows that this is not always true [8]: situations do exist where the two points of view are not equivalent, and even where the predictions of quantum mechanics for an ensemble of measurements are at variance with those obtained from an average over a phase [7]. This is not so surprising after all: the idea of a pre-existing phase is very similar to the notion of "elements of reality" [9] introduced by Einstein, Podolsky and Rosen [10]-for a double Fock state, the relative phase is nothing but what is often called a "hidden variable"-and we know that this idea combined with locality leads to the Bell theorem [1] and to contradictions with quantum mechanics. It is then natural that the notion of classical phase should also lead to Bell-type inequalities and to similar contradictions.

Such contradictions were indeed predicted in 7], but in conditions that seemed difficult to reach experimentally: precise spin measurements in $N$ separate regions of space were required ( $N$ is the total number of particles), and the numbers of results at each end of the experiment were fixed. Here we consider more realistic situations where spinless particles enter interferometers, and where the only requirement is to measure in which arm they leave them; this is accessible by laser atomic fluorescence (repeated measurements are possible in a quantum nondemolition scheme). Moreover, the number of results in each region may fluctuate freely. We study various situations involving two or three BEC's, used as sources for interferometers, and show that quantum mechanics predicts violations of the BCHSH inequalities 12], of the GHZ contradictions [13, 14] as well as of the Hardy impossibilities [15, 16]. Fock-state condensates appear as remarkably versatile, able to create violations that usually require elaborate entangled wave functions, and produce new $N$-body violations.

We first study an interferometer with a double Fockstate representing the sources, as shown in Fig.1: a condensate containing $N_{\alpha}$ particles reaches a semi-reflecting plate and is split into two coherent components $u$ and $v$; similarly, another condensate containing $N_{\beta}$ particles reaches another semi-reflecting plate and is split into two components $w$ and $t$. We assume that, in two remote regions of space $D_{A}$ and $D_{B}$, two experimenters Alice and Bob make measurements with semi-reflecting plates, recombining components $v$ and $w$ for the former, $u$ and $t$ for the latter; before the plates, they insert devices providing a phase shift, $\zeta$ for Alice, $\theta$ for Bob. We call $m_{1}$ and $m_{2}$ the number of particles that Alice detects in output 1 and 2 respectively, and $m_{3}$ and $m_{4}$ the similar quantities for Bob. Ref. [17] gives a study of a two-particle Bell inequality with this interferometer. We now calculate the 
probability $P\left(m_{1}, m_{2}, m_{3}, m_{4}\right)$ of such events.

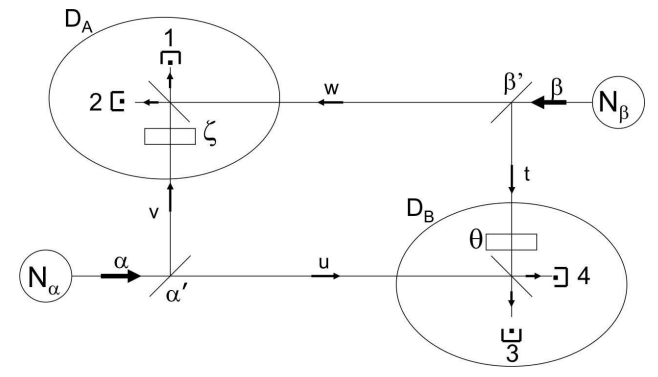

FIG. 1: Two independent condensates (populations $N_{\alpha}$ and $N_{\beta}$ ) are split into two coherent components, and then enter interferometers in two remote places $D_{A}$ and $D_{B}$; the quantum results strongly violate the $\mathrm{BCHSH}$ local realist inequalities.

The destruction operators $a_{1} \cdots a_{4}$ associated with the output modes can be written in terms of the mode operators at the sources $a_{\alpha}, a_{\beta}, a_{\alpha^{\prime}}$ and $a_{\beta^{\prime}}$ by tracing back from the detectors to the sources, with a phase shift of $\pi / 2$ at each reflection, $\zeta$ or $\theta$ at the shifters, and a $1 / \sqrt{2}$ at each beam splitter. This gives the projections of the two different source modes onto each detector mode

$$
\begin{aligned}
& a_{1}=\frac{1}{2}\left[i e^{i \zeta} a_{\alpha}+i a_{\beta}\right] \\
& a_{2}=\frac{1}{2}\left[-e^{i \zeta} a_{\alpha}+a_{\beta}\right] \\
& a_{3}=\frac{1}{2}\left[i a_{\alpha}+i e^{i \theta} a_{\beta}\right] \\
& a_{4}=\frac{1}{2}\left[a_{\alpha}-e^{i \theta} a_{\beta}\right]
\end{aligned}
$$

where we have eliminated $a_{\alpha^{\prime}}$ and $a_{\beta^{\prime}}$, which do not contribute; in short, we write these equations as $a_{i}=$ $u_{i \alpha} a_{\alpha}+u_{i \beta} a_{\beta}$. The source state is

$$
|\Phi\rangle=\frac{1}{\sqrt{N_{\alpha} ! N_{\beta} !}} a_{\alpha}^{\dagger N_{\alpha}} a_{\beta}^{\dagger N_{\beta}}|0\rangle
$$

where $a_{\alpha}^{\dagger}$ and $a_{\beta}^{\dagger}$ are creation operators and $|0\rangle$ is the vacuum. The amplitude for the system crossing all beam splitters with $m_{1} \cdots m_{4}$ particles at the detectors is:

$$
C_{m_{1}, . ., m_{4}}=\left\langle m_{1}, m_{2}, m_{3}, m_{4} \mid \Phi\right\rangle=\left\langle 0\left|\frac{a_{1}^{m_{1}} \cdots a_{4}^{m_{4}}}{\sqrt{m_{1} ! \cdots m_{4} !}} \frac{a_{\alpha}^{\dagger N_{\alpha}} a_{\beta}^{\dagger N_{\beta}}}{\sqrt{N_{\alpha} ! N_{\beta} !}}\right| 0\right\rangle
$$

We substitute (1) into this expression, and make binomial expansions of the sums $\left(u_{i \alpha} a_{\alpha}+u_{i \beta} a_{\beta}\right)^{m_{i}}$ to find

$$
C_{m_{1}, . ., m_{4}}=\frac{1}{\sqrt{N_{\alpha} ! N_{\beta} !} \sqrt{m_{1} ! \cdots m_{4} !}}\left(\prod_{i=1}^{4} \sum \frac{m_{i} !}{p_{\alpha i} ! p_{\beta i} !}\left(u_{i \alpha}\right)^{p_{\alpha i}}\left(u_{i \beta}\right)^{p_{\beta i}}\right)\left\langle 0\left|a_{\alpha}^{p_{\alpha 1}+\cdots+p_{\alpha 4}} a_{\beta}^{p_{\beta 1}+\cdots+p_{\beta 4}} a_{\alpha}^{\dagger N_{\alpha}} a_{\beta}^{\dagger N_{\beta}}\right| 0\right\rangle
$$

where the $\sum$ is a summation over $p_{\alpha i}$ and $p_{\beta i}$ with $p_{\alpha i}+p_{\beta i}=m_{i}$. The evaluation of the expectation value of the operator product gives $N_{\alpha} ! N_{\beta} ! \delta_{N_{\alpha}, p_{\alpha 1}+\cdots p_{\alpha 4}} \delta_{N_{\beta}, p_{\beta 1}+\cdots p_{\beta 4}}$, which can be included by inserting the relations $\delta_{N_{\gamma}, p_{\alpha 1}+\cdots p_{\alpha 4}}=\int_{-\pi}^{\pi} \frac{d \lambda_{\gamma}}{2 \pi} e^{i\left(p_{\gamma 1}+\cdots p_{\gamma 4}-N_{\gamma}\right) \lambda_{\gamma}}$ with $\gamma=\alpha, \beta$. If the total number of measurements $M=\sum_{i} m_{i}$ is equal to the total number of particles $N=N_{\alpha}+N_{\beta}$, the probability of obtaining result $\left(m_{1}, m_{2}, m_{3}, m_{4}\right)$ is

$$
\mathcal{P}\left(m_{1}, m_{2}, m_{3}, m_{4}\right)=\left|C_{m_{1}, \cdots m_{4}}\right|^{2}=\frac{N_{\alpha} ! N_{\beta} !}{m_{1} ! \cdots m_{4} !} \int d \tau \int d \tau^{\prime} e^{-i\left[N_{\alpha}\left(\lambda_{\alpha}-\lambda_{\alpha}^{\prime}\right)+N_{\beta}\left(\lambda_{\beta}-\lambda_{\beta}^{\prime}\right)\right]} \prod_{i=1}^{4}\left[\Omega_{i}^{\prime *} \Omega_{i}\right]^{m_{i}}
$$

with $d \tau$ representing integration over $\lambda_{\alpha}$ and $\lambda_{\beta}$ and $d \tau^{\prime}$ over the $\lambda^{\prime}$ 's, and $\Omega_{i}\left(\lambda_{a}, \lambda_{b}\right)=\left(u_{i \alpha} e^{i \lambda_{\alpha}}+u_{i \beta} e^{i \lambda_{\beta}}\right), \Omega_{i}^{\prime}=$ $\Omega_{i}\left(\lambda_{a}^{\prime}, \lambda_{b}^{\prime}\right)$. This expression simplifies with the integration variables $\lambda_{ \pm}=\left[\left(\lambda_{\alpha}+\lambda_{\alpha}^{\prime}\right) \pm\left(\lambda_{\beta}+\lambda_{\beta}^{\prime}\right)\right] / 2 ; \Lambda_{ \pm}=\left[\left(\lambda_{\alpha}-\right.\right.$ $\left.\left.\lambda_{\alpha}^{\prime}\right) \pm\left(\lambda_{\beta}-\lambda_{\beta}^{\prime}\right)\right] / 2$, since two integrations then become trivial and disappear. Using $\Lambda_{-}$parity, we then obtain

$$
\mathcal{P}\left(m_{1}, m_{2}, m_{3}, m_{4}\right) \sim \int_{-\pi}^{\pi} \frac{d \Lambda_{-}}{2 \pi} \cos \left(N_{\alpha}-N_{\beta}\right) \Lambda_{-} \int_{-\pi}^{\pi} \frac{d \lambda_{-}}{2 \pi} \prod_{i=1}^{4}\left[\cos \Lambda_{-}+\eta_{i} \cos \left(\lambda_{-}-\varphi_{i}\right)\right]^{m_{i}}
$$

where $\eta_{1}=\eta_{3}=1 ; \eta_{2}=\eta_{4}=-1 ; \varphi_{1}=\varphi_{2}=-\varsigma ; \varphi_{3}=\varphi_{4}=\theta$.

When $N=2$ it is easy to show that $\mathcal{P}(0,1,0,1)$ is equal to $\frac{1}{4} \cos ^{2}\left(\frac{\varsigma+\theta}{2}\right)$, in agreement with Ref. [17]. For any $N$, we recover the same form of the probability as for two interfering spinor condensates [7]; detectors 1 and 3 in Fig. 1 correspond to results $\eta=+1$ for spin measurements, 2 and 4 to $\eta=-1$ results. Nevertheless, instead 
of assuming that Alice and Bob measure a fixed number of spins, here the number of particles they detect can fluctuate freely, which changes the averages.

If in (6) we set $\Lambda_{-}=0$, we obtain the predictions of a pre-existing phase $\lambda_{-}$, with a product of phasedependent local probabilities $\frac{1}{2}\left[1+\eta_{i} \cos \left(\lambda_{-}-\varphi_{i}\right)\right]$ summed over all possible values of $\lambda_{-}$. But, when $\Lambda_{-}$ varies, negative "probabilities" appear in the integrand, introducing limitations to the notion of classical phase and the possibility of violations of local realism. This can happen only if all particles are measured: if $M<N$ particles are detected, summing over the unobserved results amounts to setting $\eta_{i}=0$ in the corresponding factors of (6), so that a factor $\left[\cos \Lambda_{-}\right]^{N-M}$ appears in the formula, peaked at $\Lambda_{-}=0$. Quantum violations then disappear, while the notion of relative phase re-appears.

Counting $\eta=+1$ and -1 values as above, we can define their product at Alice's location as a quantity $\mathcal{A}= \pm 1$, and at Bob's location their product as $\mathcal{B}= \pm 1$; we then have two functions to which the BCHSH theorem can be applied. The quantum average of their product is:

$$
\langle\mathcal{A B}\rangle=\sum_{m_{1} \cdots m_{4}}(-1)^{m_{2}+m_{4}} \mathcal{P}\left(m_{1}, m_{2}, m_{3}, m_{4}\right)
$$

The $m_{i}$ sums can be done, leading to an exponential of a sum of three terms that can be re-expanded in three series. If $M=N$, the four $\tau$ integrals are easy, resulting in Kronecker $\delta$ 's that collapse the sums to a single term:

$$
\langle\mathcal{A B}\rangle=\left[\cos \left(\frac{\zeta+\theta}{2}\right)\right]^{N} \delta_{N_{\alpha}, N_{\beta}}
$$

Now, the CHSH inequality tells us that:

$$
\langle\mathcal{A B}\rangle+\left\langle\mathcal{A B}^{\prime}\right\rangle+\left\langle\mathcal{A}^{\prime} \mathcal{B}\right\rangle-\left\langle\mathcal{A}^{\prime} \mathcal{B}^{\prime}\right\rangle \leq 2
$$

where letters with and without primes imply measurements at differing angles. Alice's measurement angle is taken for convenience as $\phi_{a}=\varsigma / 2$ and Bob's as $\phi_{b}=-\theta / 2$. We define $E\left(\phi_{a}-\phi_{b}\right)=\cos ^{N}\left(\phi_{a}-\phi_{b}\right)$, set $\phi_{a}-\phi_{b}=\phi_{b}-\phi_{a^{\prime}}=\phi_{b^{\prime}}-\phi_{a}=\xi$ and $\phi_{b^{\prime}}-\phi_{a^{\prime}}=3 \xi$, and maximize $Q=3 E(\xi)-E(3 \xi)$ to find the greatest violation of the inequality for each $N$. For $N=2$ we find $Q_{\max }=2.41$ in agreement with Ref. [17]; for $N=4$, $Q_{\max }=2.36$; and for $N \rightarrow \infty, Q_{\max } \rightarrow 2.32$. The system continues to violate local realism for arbitrarily large condensates. Note that every source particle must be measured, otherwise no violation is found [7].

Consider next the arrangement of Fig. 2 with three Fock condensate sources and three detector pairs; it will allow GHZ contradictions. A similar device was discussed in Ref. [18], but with only one particle per source; Ref. 14] also considered measuring spinless particles in an interferometer. We proceed as above to find a probability $P\left(m_{1} \cdots m_{6}\right)$.

For obtaining GHZ contradictions, we consider the case $N_{\alpha}=N_{\beta}=N_{\gamma}=N / 3$ and the events where all three

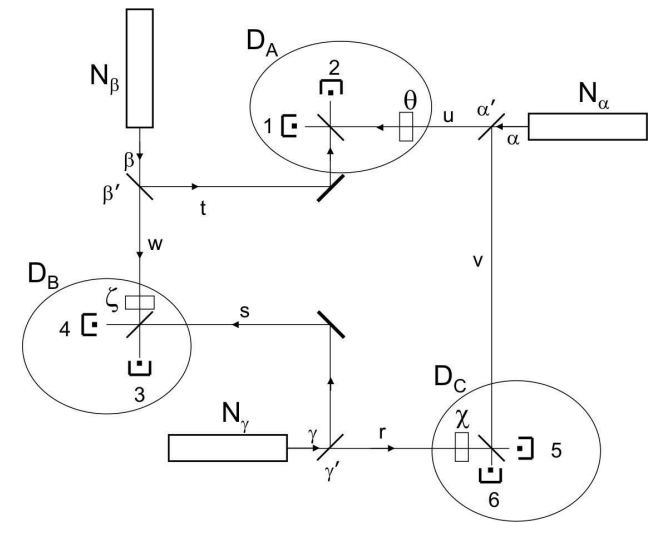

FIG. 2: Each of 3 condensates is split in 2 parts, which then enter interferometers in 3 remote places $D_{A}, D_{B}$ and $D_{C}$; the quantum results show GHZ contradictions with local realism.

detectors receive the same number of particles. Because we are considering a limited number of cases, the normalization is now different, and we must compute

$$
\begin{array}{r}
\mathcal{N} \equiv \sum_{m_{1} \cdots m_{6}} \delta_{m_{1}+m_{2}, N / 3} \times \delta_{m_{3}+m_{4}, N / 3} \\
\times \delta_{m_{5}+m_{6}, N / 3} \times \mathcal{P}\left(m_{1} \cdots m_{6}\right)
\end{array}
$$

In order to limit the sum over the $m_{i}$ 's to these cases, we introduce three more integrals over $\rho_{A}, \rho_{B}$, and $\rho_{C}$ of the form $\delta_{m_{1}+m_{2}, N / 3}=\int_{-\pi}^{\pi} \frac{d \rho_{A}}{2 \pi} e^{i\left(m_{1}+m_{2}-N / 3\right) \rho_{A}}$. To find the average $\langle\mathcal{A B C}\rangle$ for measurements done by Alice, Bob, and Carole we introduce $(-1)^{m_{2}+m_{4}+m_{6}}$ into the sums over the $m_{i}$ and perform them in the same way. Dividing by the normalization gives us

$$
\langle\mathcal{A B C}\rangle=\frac{\sum_{q}\left(\frac{N / 3 !}{(N / 3-q) ! q !}\right)^{3} e^{i(\varsigma+\theta+\chi)(N / 3-2 q)}}{\sum_{q}\left(\frac{N / 3 !}{(N / 3-q) ! q !}\right)^{3}}
$$

In the usual GHZ case $N=3$, we find $\langle\mathcal{A B C}\rangle=\cos (\varsigma+$ $\theta+\chi)$, and perfect correlations if the sum of angles is $\pi$. Local realism then gives $\langle\mathcal{A B C}\rangle=A(\varsigma) B(\theta) C(\chi)$ and

$$
\begin{aligned}
& A(\pi / 2) B(\pi / 2) C(0)=-1 \\
& A(\pi / 2) B(0) C(\pi / 2)=-1 \\
& A(0) B(\pi / 2) C(\pi / 2)=-1
\end{aligned}
$$

But then we must have $A(0) B(0) C(0)=-1$, while quantum mechanics gives +1 . For larger $N$ we also obtain contradictions, for instance when $N=9$, where

$$
\langle\mathcal{A B C}\rangle=\frac{1}{28}[27 \cos (\varsigma+\theta+\chi)+\cos 3(\varsigma+\theta+\chi)]
$$

Since both cosines change sign when the angles increase by $\pi$, the above argument remains unchanged and, again, leads to complete sign contradiction. Actually, any time $N / 3$ is odd, we get a similar result for arbitrary $N$.

Hardy impossibilities are treated by use of the interferometer shown in Fig. 3 based on the one discussed in 


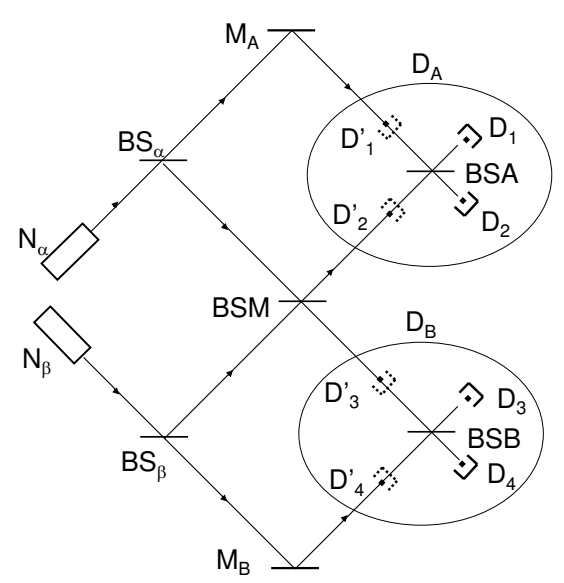

FIG. 3: An interferometer with particle sources $\alpha$ and $\beta$, beamsplitters BS and mirrors $\mathrm{M}$. In both detection regions, the detectors at $D_{i}$ may be replaced by the $D_{i}^{\prime}$, placed before the beam splitters. For appropriate path lengths and reflectivities of the beam splitters, quantum mechanics predicts the existence of events that are forbidden by local realism.

Ref. 15] for $N=2$. The heart of the system is the beam splitter at the center; due to Bose interference it has the property that, if an equal number of particles approaches each side, then an even number emerges from each side. The detection beam splitters BSA and BSB are set to have a transmission probability of $1 / 3$ and the path differences are such that, by destructive interference, no particle reaches $D_{2}$ if only source $N_{\alpha}$ is used; similarly, no particle reaches $D_{3}$ if $N_{\beta}$ alone is used. Alice can use either the detectors $D_{1,2}$ after her beam splitter, or $D_{1,2}^{\prime}$ before; Bob can choose either $D_{3,4}$, or $D_{3,4}^{\prime}$. This gives 4 arrangements of experiments: $D D, D D^{\prime}, D^{\prime} D$, or $D^{\prime} D^{\prime}$, with probability amplitudes $C_{X Y}\left(m_{1}, m_{2} ; m_{3}, m_{4}\right)$, where $X Y$ is any of these 4 arrangements and the $m$ values are the numbers of particles detected at each counter.

Assume for instance that $N=6$ and that both experimenters observe 3 particles. The probability amplitude $C_{D^{\prime} D^{\prime}}(0,3 ; 3,0)$ vanishes because of the beam splitter rule. The destructive interference effect at BSA and BSB lead to $C_{D D^{\prime}}(0,3 ; 1,2)=C_{D D^{\prime}}(0,3 ; 2,1)=$ $C_{D D^{\prime}}(0,3 ; 0,3)=0$; but $C_{D D^{\prime}}(0,3 ; 3,0) \neq 0$. Thus, if Alice observes 3 particles at $D_{2}$, when Bob uses the primed detectors he observes with certainty 3 particles at $D_{3}^{\prime}$; similarly, if Bob has seen 3 particles in $D_{3}$, in the $D^{\prime} D$ configuration Alice must see 3 in $D_{2}^{\prime}$.

If both do unprimed experiments, we find $C_{D D}(0,3 ; 3,0)=1 / 216$, which shows that events exist where 3 particles are detected at both detectors $D_{2}$ and $D_{3}$. In any of these events, if Bob had at the last instant changed to the primed detectors, he would surely have obtained three particles in $D_{3}^{\prime}$, because of the certainty mentioned above; if Alice had changed detectors instead of Bob, she would have obtained 3 particles in $D_{2}^{\prime}$. Now, had both changed their minds after the emission and chosen the primed arrangement, local realism implies that they would have found 3 particles in each $D_{2}^{\prime}$ and $D_{3}^{\prime}$ : such events must exist. But its quantum probability is exactly zero, in complete contradiction. This argument can be generalized to all cases of odd $N / 2$ emitted from each source.

In conclusion, we think that the answer to Anderson's question is: "In most cases, this view leads to correct quantum predictions, but not always." It is sufficient for instance in the situations described in [2]; but when all particles are measured, quantum mechanics sometimes predicts probabilities that cannot be explained in terms of a pre-existing phase, and reveal a more fundamental quantum character of this physical quantity. This creates the possibility of new $N$-body violations of local realism with the use of Fock-state condensates.

We thank W.D. Phillips for useful discussions. Laboratoire Kastler Brossel is "UMR 8552 du CNRS, de l'ENS, et de l'Université Pierre et Marie Curie".

[1] J.E. Simsarian, J. Denschlag, M. Edwards, C.W. Clark, L. Deng, E.W. Hagley, K. Helmerson, S.L. Rolston and W.D. Phillips, Phys. Rev. Lett. 85, 2040 (2000).

[2] M.R. Andrews, C.G. Townsend, H.-J. Miesner, D.S. Durfee, D.M. Kurn, W. Ketterle, Science, 275, 637 (1997).

[3] P.W. Anderson, in The Lesson of Quantum Theory, eds. J. de Boer, E. Dahl, and O. Ulfbeck (Elsevier, New York, 1986).

[4] J. Javanainen and Sung Mi Yoo, Phys. Rev. Lett. 76, 161-164 (1996).

[5] Y. Castin and J. Dalibard, Phys. Rev. A 55, 4330 (1997).

[6] M.H. Wheeler, K.M. Mertes, J.D. Erwin and D.S. Hall, Phys. Rev. Lett 93, 170402 (2004).

[7] F. Laloë and W.J. Mullin, Phys. Rev. Lett. 99, 150401 (2007); Phys. Rev. A, 77, 022108 (2008).

[8] A.J. Leggett and F. Sols, Found. Phys. 21, 353-364 (1991); A.J. Leggett, in Bose-Einstein condensation, eds. A. Griffin, D.W. Snoke and S. Stringari, Cambridge University Press (1995).

[9] F. Laloë, Europ. Phys. J. D, 33, 87 (2005); see also cond-mat/0611043.

[10] A. Einstein, B. Podolsky and N. Rosen, Phys. Rev. 47, 777 (1935).

[11] J.S. Bell, Physics 1, 195 (1964), reprinted in J.S. Bell, Speakable and unspeakable in quantum mechanics, (Cambridge University Press, Cambridge, 1987).

[12] J.F. Clauser, M.A. Horne, A. Shimony and R.A. Holt, Phys. Rev. Lett. 23, 880 (1969).

[13] D.M. Greenberger, M.A. Horne and A. Zeilinger, in Bell theorem, quantum theory, and conceptions of the universe, ed. M. Kafatos, (Kluwer, 1989) page 74.

[14] Greenberger, M.A. Horne, A. Shimony and A. Zeilinger, Am. J. Phys. 58, 1131 (1990).

[15] L. Hardy, Phys. Lett. A 167, 17-23 (1992).

[16] L. Hardy, Phys. Rev. Lett. 71, 1665 (1993).

[17] B. Yurke and D. Stoler, Phys. Rev. A 46, 2229 (1992).

[18] B. Yurke and D. Stoler, Phys. Rev. Lett 68, 1251 (1992). 
